\title{
Coincidence Points of Weaker Contractions in Partially Ordered Metric Spaces
}

\author{
Kuo-Ching Jen, ${ }^{1}$ Ing-Jer Lin, ${ }^{2}$ and Chi-Ming Chen ${ }^{3}$ \\ ${ }^{1}$ General Education Center, St. John's University, Taiwan \\ ${ }^{2}$ Department of Mathematics, National Kaohsiung Normal University, Kaohsiung 824, Taiwan \\ ${ }^{3}$ Department of Applied Mathematics, National Hsinchu University of Education, Taiwan
}

Correspondence should be addressed to Chi-Ming Chen; ming@mail.nhcue.edu.tw

Received 6 June 2013; Accepted 4 August 2013

Academic Editor: E. Karapinar

Copyright (C) 2013 Kuo-Ching Jen et al. This is an open access article distributed under the Creative Commons Attribution License, which permits unrestricted use, distribution, and reproduction in any medium, provided the original work is properly cited.

We prove new coincidence point theorems for the $(\varphi, \psi, \phi, \xi)$-contractions and generalized Meir-Keeler-type $\alpha$ - $\psi$-contractions in partially ordered metric spaces. Our results generalize many recent coincidence point theorems in the literature.

\section{Introduction and Preliminaries}

Throughout this paper, by $\mathbb{R}^{+}$, we denote the set of all nonnegative real numbers, while $\mathbb{N}$ is the set of all natural numbers. Let $(X, d)$ be a metric space, $D$ a subset of $X$, and $f: D \rightarrow X$ a map. We say $f$ is contractive if there exists $\alpha \in[0,1)$ such that for all $x, y \in D$,

$$
d(f x, f y) \leq \alpha \cdot d(x, y)
$$

The well-known Banach's fixed point theorem asserts that if $D=X, f$ is contractive and $(X, d)$ is complete, then $f$ has a unique fixed point in $X$. It is well known that the Banach contraction principle [1] is a very useful and classical tool in nonlinear analysis. Also, this principle has many generalizations. For instance, a mapping $f: X \rightarrow X$ is called a quasicontraction if there exists $k<1$ such that

$$
\begin{aligned}
d(f x, f y) \leq k \cdot \max & \{d(x, y), d(x, f x), \\
& d(y, f y), d(x, f y), d(y, f x)\},
\end{aligned}
$$

for any $x, y \in X$. In 1974, Cirić [2] introduced these maps and proved an existence and uniqueness fixed point theorem.

Recently, Eslamian and Abkar proved the following theorem.
Theorem 1 (see [3]). Let $(X, d)$ be a complete metric space and $f: X \rightarrow X$ be such that

$$
\begin{array}{r}
\psi(d(f x, f y)) \leq \alpha(d(x, y))-\beta(d(x, y)) \\
\text { for each } x, y \in X
\end{array}
$$

where $\psi, \alpha, \beta: \mathbb{R}^{+} \rightarrow \mathbb{R}^{+}$are as follows: $\psi$ is continuous and nondecreasing, $\alpha$ is continuous, $\beta$ is lower semicontinuous, and

$$
\begin{gathered}
\psi(t)-\alpha(t)+\beta(t)>0 \quad \forall t>0, \\
\psi(t)=0 \quad \text { iff } t=0, \quad \alpha(0)=\beta(0)=0 .
\end{gathered}
$$

Then $f$ has a fixed point in $X$.

Recently, fixed point theory has developed rapidly in partially ordered metric spaces (e.g., [4-8]).

In 2012, Choudhury and Kundu [9] proved the following coincidence theorem as a generalization of Theorem 1 .

Theorem 2 (see [9]). Let $(X, \sqsubseteq$ ) be a partially ordered set and suppose that there exists a metric $d$ in $X$ such that $(X, d)$ is a complete metric space and $f, g: X \rightarrow X$ be such that $f X \subset$ $g X, f$ is $g$-nondecreasing, $g X$ is closed, and

$$
\begin{array}{r}
\psi(d(f x, f y)) \leq \alpha(d(g x, g y))-\beta(d(g x, g y)), \\
\text { for each } x, y \in X \text { such that } g x \sqsubseteq g y,
\end{array}
$$


where $\psi, \alpha, \beta: \mathbb{R}^{+} \rightarrow \mathbb{R}^{+}$are such that $\psi$ is continuous and nondecreasing, $\alpha$ is continuous, $\beta$ is lower semicontinuous, and

$$
\begin{gathered}
\psi(t)-\alpha(t)+\beta(t)>0 \quad \forall t>0, \\
\psi(t)=0 \quad \text { iff } t=0, \quad \alpha(0)=\beta(0)=0 .
\end{gathered}
$$

Also, if any nondecreasing sequence $\left\{x_{n}\right\}$ in $X$ converges to $\nu$, then we assume that

$$
x_{n} \sqsubseteq \nu \quad \forall n \in \mathbb{N} .
$$

If there exists $x_{0} \in X$ with $g x_{0} \sqsubseteq f x_{0}$, then $f$ and $g$ have $a$ coincidence point in $X$.

In this paper, we prove new coincidence point theorems for the $(\varphi, \psi, \phi, \xi)$-contractions and generalized Meir-Keelertype $\alpha-\psi$-contractions in partially ordered metric spaces. Our results generalize many recent coincidence point theorems in the literature.

\section{Main Results}

We start with the following definition.

Definition 3 ( $g$-nondecreasing mapping [4]). Let $(X, \sqsubseteq$ ) be a partially ordered set and $f, g: X \rightarrow X$. Then $f$ is said to be $g$-nondecreasing if, for $x, y \in X$,

$$
g x \sqsubseteq g y \Longrightarrow f x \sqsubseteq f y .
$$

In the sequel, we denote by $\Psi$ the class of functions $\psi$ : $\mathbb{R}^{+5} \rightarrow \mathbb{R}^{+}$satisfying the following conditions:

$\left(\psi_{1}\right) \psi$ is an increasing, continuous function in each coordinate,

$\left(\psi_{2}\right)$ for all $t \in \mathbb{R}^{+}, \psi(t, t, t, 0,2 t) \leq t, \psi(t, t, t, 2 t, 0) \leq t$, $\psi(0,0, t, t, 0) \leq t$, and $\psi(t, 0,0, t, t) \leq t$,

$\left(\psi_{3}\right) \psi\left(t_{1}, t_{2}, t_{3}, t_{4}, t_{5}\right)=0$ if and only if $t_{1}=t_{2}=t_{3}=t_{4}=$ $t_{5}=0$.

Next, we denote by $\Phi$ the class of functions $\phi: \mathbb{R}^{+} \rightarrow \mathbb{R}^{+}$ satisfying the following conditions:

$\left(\phi_{1}\right) \phi$ is a continuous function and monotone nondecreasing;

$\left(\phi_{2}\right) \phi(t)>0$ for $t>0$ and $\phi(0)=0$;

$\left(\phi_{3}\right) \phi$ is subadditive, that is, $\phi\left(t_{1}+t_{2}\right) \leq \phi\left(t_{1}\right)+\phi\left(t_{2}\right)$ for all $t_{1}, t_{2}>0$.

And, we denote the following sets of functions:

$$
\Theta=\left\{\varphi: \mathbb{R}^{+} \longrightarrow \mathbb{R}^{+} \text {such that } \varphi \text { is continuous }\right\},
$$$$
\Xi=\left\{\xi: \mathbb{R}^{+} \longrightarrow \mathbb{R}^{+} \text {such that } \xi \text { is lower continuous }\right\} \text {. }
$$

Let $X$ be a nonempty set and $(X, \sqsubseteq)$ be a partially ordered set endowed with a metric $d$. Then, the triple $(X, \sqsubseteq, d)$ is called a partially ordered metric space.

We now state the $(\varphi, \psi, \phi, \xi)$-contraction and the main fixed point theorem for the $(\varphi, \psi, \phi, \xi)$-contraction in partially ordered metric spaces, as follows.
Definition 4. Let $(X, \sqsubseteq, d)$ be a partially ordered complete metric space, and let $f, g: X \rightarrow X$. Then the pair $(f, g)$ is called a $(\varphi, \psi, \phi, \xi)$-contraction if the following inequality holds:

$$
\begin{aligned}
\varphi(d(f x, f y)) \\
\leq \psi(\phi(d(g x, g y)), \phi(d(g x, f x)), \\
\quad \phi(d(g y, f y)), \phi(d(g x, f y)), \phi(d(g y, f x))) \\
-\xi(\max \{d(g x, g y), d(g x, f x), d(g y, f y)\}),
\end{aligned}
$$

for all $x, y \in X$ with $g x \sqsubseteq g y$, where $\varphi \in \Theta, \psi \in \Psi, \phi \in \Phi$ and $\xi \in \Xi$.

We now state the main fixed point theorem for the $(\varphi, \psi, \phi, \xi)$-contraction in partially ordered metric spaces, as follows.

Theorem 5. Let $(X, \sqsubseteq, d)$ be a partially ordered complete metric space, and let $f, g: X \rightarrow X$ be such that $f X \subset g X, f$ is $g$-nondecreasing and $g X$ is closed. Suppose the pair $(f, g)$ is $a(\varphi, \psi, \phi, \xi)$-contraction, and

$$
\begin{gathered}
\varphi(t)-\phi(t)+\xi(t)>0 \quad \forall t>0, \\
\varphi(t)=0 \quad \text { iff } t=0, \quad \phi(0)=\xi(0)=0 .
\end{gathered}
$$

Also, if any nondecreasing sequence $\left\{x_{n}\right\}$ in $X$ converges to $v$, then we assume that

$$
x_{n} \sqsubseteq \nu \quad \forall n \in \mathbb{N} .
$$

If there exists $x_{0} \in X$ with $g x_{0} \sqsubseteq f x_{0}$, then $f$ and $g$ have $a$ coincidence point in $X$.

Proof. Since $f X \subset g X$ and there exists $x_{0} \in X$ with $g x_{0} \sqsubseteq$ $f x_{0}$, we can choose $x_{1} \in X$ such that $g x_{1}=f x_{0}$. Since $f$ is $g$-nondecreasing, we have $f x_{0} \sqsubseteq f x_{1}$. In this process, we construct the sequence $\left\{x_{n}\right\}$ recursively as

$$
f x_{n}=g x_{n+1} \quad \forall n \in \mathbb{N} .
$$

Thus, we also conclude that

$$
\begin{aligned}
g x_{0} \sqsubseteq f x_{0} & =g x_{1} \sqsubseteq f x_{1}=g x_{2} \sqsubseteq \cdots \sqsubseteq f x_{n-1} \\
& =g x_{n} \sqsubseteq f x_{n}=g x_{n+1} \sqsubseteq \cdots
\end{aligned}
$$

If any two consecutive terms in (14) are equal, then the conclusion of the theorem follows. So we may assume that

$$
d\left(f x_{n-1}, f x_{n}\right) \neq 0, \quad \forall n \in \mathbb{N} .
$$

Now, we claim that $d\left(f x_{n}, f x_{n+1}\right) \leq d\left(f x_{n-1}, f x_{n}\right)$ for all $n \in$ $\mathbb{N}$. If not, we assume that $d\left(f x_{n-1}, f x_{n}\right)<d\left(f x_{n}, f x_{n+1}\right)$ for 
some $n \in \mathbb{N}$, substituting $x=x_{n}$ and $y=x_{n+1}$ in (10) and using the definition of the function $\psi$, we have

$$
\begin{gathered}
\psi\left(\phi\left(d\left(g x_{n}, g x_{n+1}\right)\right), \phi\left(d\left(g x_{n}, f x_{n}\right)\right),\right. \\
\phi\left(d\left(g x_{n+1}, f x_{n+1}\right)\right), \phi\left(d\left(g x_{n}, f x_{n+1}\right)\right), \\
\left.\phi\left(d\left(g x_{n+1}, f x_{n}\right)\right)\right) \\
=\psi\left(\phi\left(d\left(f x_{n-1}, f x_{n}\right)\right), \phi\left(d\left(f x_{n-1}, f x_{n}\right)\right),\right. \\
\phi\left(d\left(f x_{n}, f x_{n+1}\right)\right), \phi\left(d\left(f x_{n-1}, f x_{n+1}\right)\right), \\
\left.\quad \phi\left(d\left(f x_{n}, f x_{n}\right)\right)\right) \\
\leq \psi\left(\phi\left(d\left(f x_{n}, f x_{n+1}\right)\right), \phi\left(d\left(f x_{n}, f x_{n+1}\right)\right),\right. \\
\left.\phi\left(d\left(f x_{n}, f x_{n+1}\right)\right), 2 \phi\left(d\left(f x_{n}, f x_{n+1}\right)\right), \phi(0)\right) \\
\leq \phi\left(d\left(f x_{n}, f x_{n+1}\right)\right), \\
\xi\left(\operatorname { m a x } \left\{d\left(g x_{n}, g x_{n+1}\right), d\left(g x_{n}, f x_{n}\right),\right.\right. \\
\left.\left.d\left(g x_{n+1}, f x_{n+1}\right)\right\}\right) \\
=\xi\left(d\left(f x_{n}, f x_{n+1}\right)\right), \\
\quad \max _{\{}\left\{d\left(f x_{n-1}, f x_{n}\right), d\left(f x_{n-1}, f x_{n}\right),\right. \\
\left.\left.d\left(f x_{n}, f x_{n+1}\right)\right\}\right)
\end{gathered}
$$

and hence

$$
\varphi\left(d\left(f x_{n}, f x_{n+1}\right)\right) \leq \phi\left(d\left(f x_{n}, f x_{n+1}\right)\right)-\xi\left(d\left(f x_{n}, f x_{n+1}\right)\right) .
$$

Since $\varphi(t)-\phi(t)+\xi(t)>0$ for all $t>0$, we have that $d\left(f x_{n}, f x_{n+1}\right)=0$, which contradicts to (15). Therefore, we conclude that

$$
d\left(f x_{n}, f x_{n+1}\right) \leq d\left(f x_{n-1}, x_{n}\right) \quad \forall n \in \mathbb{N} .
$$

From above argument, we also have that for each $n \in \mathbb{N}$

$$
\varphi\left(d\left(f x_{n}, f x_{n+1}\right)\right) \leq \phi\left(d\left(f x_{n-1}, f x_{n}\right)\right)-\xi\left(d\left(f x_{n-1}, f x_{n}\right)\right) .
$$

It follows (18) that the sequence $\left\{d\left(f x_{n}, f x_{n+1}\right)\right\}$ is monotone decreasing, it must converge to some $\eta \geq 0$. Taking limit as $n \rightarrow \infty$ in (19) and using the continuities of $\varphi$ and $\phi$ and the lower semicontinuity of $\xi$, we get

$$
\varphi(\eta) \leq \phi(\eta)-\xi(\eta)
$$

which implies that $\eta=0$. So we conclude that

$$
\lim _{n \rightarrow \infty} d\left(f x_{n}, f x_{n+1}\right)=0 .
$$

We next claim that $\left\{f x_{n}\right\}$ is a Cauchy sequence, that is, for every $\varepsilon>0$, there exists $n \in \mathbb{N}$ such that if $p, q \geq n$, then $d\left(f x_{p}, f x_{q}\right)<\varepsilon$.

Suppose the above statement is false. Then there exists $\epsilon>$ 0 such that for any $n \in \mathbb{N}$, there are $p_{n}, q_{n} \in \mathbb{N}$ with $p_{n}>q_{n} \geq$ $n$ satisfying

$$
d\left(f x_{q_{n}}, f x_{p_{n}}\right) \geq \epsilon .
$$

Further, corresponding to $q_{n} \geq n$, we can choose $p_{n}$ in such a way that it is the smallest integer with $p_{n}>q_{n} \geq n$ and $d\left(f x_{q_{n}}, f x_{p_{n}}\right) \geq \epsilon$. Therefore $d\left(f x_{q_{n}}, f x_{p_{n}-1}\right)<\epsilon$. Now we have that for all $n \in \mathbb{N}$

$$
\begin{aligned}
\epsilon & \leq d\left(f x_{p_{n}}, f x_{q_{n}}\right) \\
& \leq d\left(f x_{p_{n}}, f x_{p_{n}-1}\right)+d\left(f x_{p_{n}-1}, f x_{q_{n}}\right) \\
& <d\left(f x_{p_{n}}, f x_{p_{n}-1}\right)+\epsilon .
\end{aligned}
$$

Letting $n \rightarrow \infty$, then we get

$$
\lim _{n \rightarrow \infty} d\left(f x_{p_{n}}, f x_{q_{n}}\right)=\epsilon .
$$

On the other hand, we have

$$
\begin{aligned}
d\left(f x_{p_{n}}, f x_{q_{n}}\right) \leq & d\left(f x_{p_{n}}, f x_{p_{n}-1}\right)+d\left(f x_{p_{n}-1}, f x_{q_{n}-1}\right) \\
& +d\left(f x_{q_{n}-1}, f x_{q_{n}}\right), \\
d\left(f x_{p_{n}-1}, f x_{q_{n}-1}\right) \leq & d\left(f x_{p_{n}-1}, f x_{p_{n}}\right)+d\left(f x_{p_{n}}, f x_{q_{n}}\right) \\
+ & d\left(f x_{q_{n}}, f x_{q_{n}-1}\right) .
\end{aligned}
$$

Letting $n \rightarrow \infty$, then we get

$$
\lim _{n \rightarrow \infty} d\left(f x_{p_{n}-1}, f x_{q_{n}-1}\right)=\epsilon .
$$

By (14), we have that the elements $g x_{p_{n}}$ and $g x_{q_{n}}$ are comparable. Substituting $x=x_{p_{n}}$ and $y=x_{q_{n}}$ in (10), we have that for all $n \in \mathbb{N}$,

$$
\begin{gathered}
\psi\left(\phi\left(d\left(g x_{p_{n}}, g x_{q_{n}}\right)\right), \phi\left(d\left(g x_{p_{n}}, f x_{p_{n}}\right)\right),\right. \\
\phi\left(d\left(g x_{q_{n}}, f x_{q_{n}}\right)\right), \phi\left(d\left(g x_{p_{n}}, f x_{q_{n}}\right)\right), \\
\left.\phi\left(d\left(g x_{q_{n}}, f x_{p_{n}}\right)\right)\right) \\
\leq \psi\left(\phi\left(d\left(f x_{p_{n}-1}, f x_{q_{n}-1}\right)\right), \phi\left(d\left(f x_{p_{n}-1}, f x_{p_{n}}\right)\right),\right. \\
\phi\left(d\left(f x_{q_{n}-1}, f x_{q_{n}}\right)\right), \phi\left(d\left(f x_{p_{n}-1}, f x_{q_{n}}\right)\right), \\
\left.\phi\left(d\left(f x_{q_{n}-1}, f x_{p_{n}}\right)\right)\right) \\
\phi\left(d\left(f x_{p_{n}-1}, f x_{q_{n}-1}\right)\right), \phi\left(d\left(f x_{p_{n}-1}, f x_{p_{n}}\right)\right), \\
\left.+\phi\left(f x_{q_{n}-1}, f x_{q_{n}}\right)\right), \phi\left(d\left(f x_{p_{n}-1}, f x_{p_{n}}\right)\right) \\
+\phi\left(d\left(f x_{p_{n}}, f x_{q_{n}}\right)\right), \phi\left(d\left(f x_{q_{n}-1}, f x_{q_{n}}\right)\right) \\
\left.\left.M\left(x_{p_{n}}\right)\right)\right), \\
\left.\quad x_{q_{n}}\right)=\max \left\{d\left(g x_{p_{n}}, g x_{q_{n}}\right),\right. \\
\left.d\left(g x_{p_{n}}, f x_{p_{n}}\right), d\left(g x_{q_{n}}, f x_{q_{n}}\right)\right\} \\
=\max \left\{d\left(f x_{p_{n}-1}, f x_{q_{n}-1}\right), d\left(f x_{p_{n}-1}, f x_{p_{n}}\right),\right. \\
\left.\left.\phi x_{q_{n}-1}, f x_{q_{n}}\right)\right\} . \\
\phi(d)
\end{gathered}
$$


By above argument and using inequality (10), we can conclude that

$$
\begin{aligned}
\varphi(\epsilon) & \leq \psi(\phi(\epsilon), 0,0, \phi(\epsilon), \phi(\epsilon))-\xi(\epsilon) \\
& \leq \phi(\epsilon)-\xi(\epsilon),
\end{aligned}
$$

which implies that $\epsilon=0$, a contradiction. Therefore, the sequence $\left\{f x_{n}\right\}$ is a Cauchy sequence.

Since $X$ is complete and $g X$ is closed, there exists $\nu \in X$ such that

$$
\lim _{n \rightarrow \infty} g x_{n}=\lim _{n \rightarrow \infty} f x_{n}=g \nu .
$$

Later, we prove that $v$ is a coincidence point of $f$ and $g$. From (14) and (29), we deduce that

$$
g x_{n} \sqsubseteq g v, \quad \forall n \in \mathbb{N} .
$$

Substituting $x=x_{n}$ and $y=v$ in (10), we have that

$$
\begin{aligned}
& \varphi\left(d\left(f x_{n}, f v\right)\right) \\
& \leq \psi\left(\phi\left(d\left(g x_{n}, g \nu\right)\right), \phi\left(d\left(g x_{n}, f x_{n}\right)\right),\right. \\
& \phi(d(g \nu, f v)), \phi\left(d\left(g x_{n}, f v\right)\right), \\
& \left.\quad \phi\left(d\left(g \nu, f x_{n}\right)\right)\right) \\
& -\xi\left(\max \left\{d\left(g x_{n}, g \nu\right), d\left(g x_{n}, f x_{n}\right), d(g \nu, f v)\right\}\right) .
\end{aligned}
$$

Taking $n \rightarrow \infty$ in the above inequality, we have

$$
\begin{aligned}
\varphi(d(g \nu, f v)) \leq & \psi(0,0, \phi(d(g \nu, f v)), \phi(d(g \nu, f v)), 0) \\
& -\xi(d(g \nu, f v)) \leq \phi(d(g \nu, f v)) \\
& -\xi(d(g \nu, f v)),
\end{aligned}
$$

which implies that $d(g v, f v)=0$, that is, $g v=f v$. So we complete the proof.

We give the following example to illustrate Theorem 5 .

Example 6. Let $X=[0,1]$. We define a partial order “ $\sqsubseteq$ " on $X$ as $x \sqsubseteq y$ if and only if $x \geq y$ for all $x, y \in X$. We take the usual metric $d(x, y)=|x-y|$ for all $x, y \in X$. Let $f, g: X \rightarrow X$ be defined as

$$
f(x)=\frac{1}{16} x^{2}, \quad g(x)=\frac{1}{4} x^{2} .
$$

Let $\varphi, \phi, \xi: \mathbb{R}^{+} \rightarrow \mathbb{R}^{+}$be defined as

$$
\varphi(t)=\phi(t)=t, \quad \xi(t)=\frac{t}{8} \quad \forall t \in[0,1],
$$

and let $\psi: \mathbb{R}^{+5} \rightarrow \mathbb{R}^{+}$denote

$$
\psi\left(t_{1}, t_{2}, t_{3}, t_{4}, t_{5}\right)=\frac{1}{2} \cdot \max \left\{t_{1}, t_{2}, t_{3}, \frac{t_{4}}{2}, \frac{t_{5}}{2}\right\} .
$$

Without loss of generality, we assume that $x>y$ and verity inequality (10).

For all $x, y \in[0,1]$ with $x>y$, we have

$$
\begin{aligned}
& \varphi(d(f x, f y))=\frac{1}{16}\left(x^{2}-y^{2}\right), \\
& \phi(d(g x, g y))=\frac{1}{4}\left(x^{2}-y^{2}\right), \\
& \phi(d(g x, f x))=\frac{1}{4} x^{2}-\frac{1}{16} x^{2}=\frac{3}{16} x^{2}, \\
& \phi(d(g y, f y))=\frac{1}{4} y^{2}-\frac{1}{16} y^{2}=\frac{3}{16} y^{2}, \\
& \phi(d(g x, f y))=\frac{1}{4} x^{2}-\frac{1}{16} y^{2}>\frac{3}{16} x^{2}, \\
& \phi(d(f x, g y))=\left|\frac{1}{16} x^{2}-\frac{1}{4} y^{2}\right|, \\
& \xi(\max d(g x, g y), d(g x, f x), d(g y, f y)) \\
& = \begin{cases}\frac{1}{4}\left(x^{2}-y^{2}\right), & \text { if } x>2 y, \\
\frac{3}{16} x^{2}, & \text { if } x \leq 2 y,\end{cases} \\
& \psi(\phi(d(g x, g y)), \phi(d(g x, f x)), \phi(d(g y, f y)), \\
& \phi(d(g x, f y)), \phi(d(f x, g y))) \\
& =\frac{1}{8} x^{2}-\frac{1}{32} y^{2} \text {. }
\end{aligned}
$$

Therefore, inequality (10) is satisfied and all the conditions of Theorem 5 are satisfied, and we obtained that 0 is a coincidence point of $f$ and $g$. let

Applying Definition 4, Theorem 5, and Example 6, if we

$$
\begin{gathered}
\psi(\phi(d(g x, g y)), \phi(d(g x, f x)), \phi(d(g y, f y)), \\
\phi(d(g x, f y)), \phi(d(g y, f x))) \\
=\max \{\phi(d(g x, g y)), \phi(d(g x, f x)), \phi(d(g y, f y)), \\
\frac{1}{2} \phi(d(g x, f y)), \frac{1}{2} \phi(d(f x, g y)\},
\end{gathered}
$$

we are easy to get the following theorem.

Theorem 7. Let $(X, \sqsubseteq, d)$ be a partially ordered complete metric space, and let $f, g: X \rightarrow X$ be such that $f X \subset g X, f$ is $g$-nondecreasing, $g X$ is closed, and

$$
\begin{aligned}
& \varphi(d(f x, f y)) \\
& \leq \max \{\phi(d(g x, g y)), \phi(d(g x, f x)), \\
& \quad \phi(d(g y, f y)), \phi(d(g x, f y)), \psi(d(f x, g y))\} \\
& -\xi(\max \{d(g x, g y), d(g x, f x), d(g y, f y)\}),
\end{aligned}
$$


for all $x, y \in X$ such that $g x \sqsubseteq g y$, where $\varphi \in \Theta, \psi \in \Psi, \phi \in \Phi$ and $\xi \in \Xi$, and

$$
\begin{gathered}
\varphi(t)-\phi(t)+\xi(t)>0 \quad \forall t>0, \\
\varphi(t)=0 \quad \text { iff } t=0, \quad \phi(0)=\xi(0)=0 .
\end{gathered}
$$

Also, if any nondecreasing sequence $\left\{x_{n}\right\}$ in $X$ converges to $v$, then one assumes that

$$
x_{n} \sqsubseteq \nu \quad \forall n \in \mathbb{N} .
$$

If there exists $x_{0} \in X$ with $g x_{0} \sqsubseteq f x_{0}$, then $f$ and $g$ have a coincidence point in $X$.

In the other research of this paper, we recall the MeirKeeler-type contraction [10] and $\alpha$-admissible mapping [11]. In 1969, Meir and Keeler [10] introduced the following notion of Meir-Keeler-type contraction in a metric space $(X, d)$.

Definition 8 . Let $(X, d)$ be a metric space, $f: X \rightarrow X$. Then $f$ is called a Meir-Keeler-type contraction whenever for each $\eta>0$ there exists $\gamma>0$ such that

$$
\eta \leq d(x, y)<\eta+\gamma \Longrightarrow d(f x, f y)<\eta .
$$

And, the following definition was introduced in [11].

Definition 9. Let $f: X \rightarrow X$ be a self-mapping of a set $X$ and $\alpha: X \times X \rightarrow \mathbb{R}^{+}$. Then $f$ is called a $\alpha$-admissible mapping if

$$
x, y \in X, \quad \alpha(x, y) \geq 1 \Longrightarrow \alpha(f x, f y) \geq 1 .
$$
follows.

We introduce the notion of $\alpha$-g-admissible mapping, as

Definition 10. Let $f, g: X \rightarrow X$ be a self-mapping of a set $X$ and $\alpha: X \times X \rightarrow \mathbb{R}^{+}$. Then $f$ is called a $\alpha$ - $g$-admissible mapping if

$$
x, y \in X, \quad \alpha(g x, g y) \geq 1 \Longrightarrow \alpha(f x, f y) \geq 1 .
$$

We give the following example to illustrate Definition 10. Example 11. Let $X=\mathbb{R}^{+}$and we define

$$
\begin{gathered}
g(x)=x+\frac{1}{2}, \quad f(x)=g(x)+\frac{1}{x+1}, \\
\alpha(x, y)=x+y .
\end{gathered}
$$

Then $f$ is a $\alpha$-g-admissible mapping.

We now state the new notions of generalized Meir-Keelertype $\psi$-contractions and generalized Meir-Keeler-type $\alpha$ - $\psi$ contractions in partially ordered complete metric spaces, as follows.

Definition 12 . Let $(X, \sqsubseteq, d)$ be a partially ordered complete metric space, and let $f, g: X \rightarrow X$. Then the pair $(f, g)$ is called a generalized Meir-Keeler-type $\psi$-contraction whenever for each $\eta>0$, there exists $\delta>0$ such that

$$
\begin{gathered}
\eta \leq \psi(d(g x, g y), d(g x, f x), d(g y, f y), \\
d(g x, f y), d(g y, f x)) \\
<\eta+\delta \Longrightarrow d(f x, f y)<\eta,
\end{gathered}
$$

for all $x, y \in X$ with $g x \sqsubseteq g y$, where $\psi \in \Psi$.
Definition 13. Let $(X, \sqsubseteq, d)$ be a partially ordered complete metric space, let $f, g: X \rightarrow X$, and $\alpha: X \times X \rightarrow$ $\mathbb{R}^{+}$. Then $(f, g)$ is called a generalized Meir-Keeler-type $\alpha$ $\psi$-contraction if the following conditions hold:

(1) $f$ is $\alpha$-g-admissible;

(2) for each $\eta>0$ there exists $\delta>0$ such that

$$
\begin{aligned}
\eta \leq \psi & (d(g x, g y), d(g x, f x), d(g y, f y), \\
& d(g x, f y), d(g y, f x)) \\
<\eta+\delta \Longrightarrow & \alpha(f x, f x) \alpha(g y, g y) d(f x, f y)<\eta,
\end{aligned}
$$

for all $x, y \in X$ with $g x \sqsubseteq g y$, where $\psi \in \Psi$.

Remark 14. Note that if $f$ is a generalized Meir-Keeler-type $\alpha-\psi$-contraction, then we have that for all $x, y \in X$

$$
\begin{gathered}
\alpha(f x, f x) \alpha(g y, g y) d(f x, f y) \\
\leq \psi(d(g x, g y), d(g x, f x), d(g y, f y), \\
d(g x, f y), d(g y, f x)) .
\end{gathered}
$$

Further, if

$$
\begin{gathered}
\psi(d(g x, g y), d(g x, f x), d(g y, f y), \\
d(g x, f y), d(g y, f x))=0,
\end{gathered}
$$

then $d(f x, f y)=0$.

On the other hand, if

$$
\begin{gathered}
\psi(d(g x, g y), d(g x, f x), d(g y, f y), \\
d(g x, f y), d(g y, f x))>0,
\end{gathered}
$$

then

$$
\begin{gathered}
\alpha(f x, f x) \alpha(g y, g y) d(f x, f y) \\
<\psi(d(g x, g y), d(g x, f x), d(g y, f y), \\
d(g x, f y), d(g y, f x)) .
\end{gathered}
$$

We now state our main result for the generalized MeirKeeler-type $\alpha$ - $\psi$-contraction, as follows.

Theorem 15. Let $(X, \sqsubseteq, d)$ be a partially ordered complete metric space, let $\alpha: X \times X \rightarrow \mathbb{R}^{+}$be continuous in each coordinate, and let $f, g: X \rightarrow X$ be such that $f X \subset$ $g X, f$ is $g$-nondecreasing, and $g X$ is closed. Suppose the pair $(f, g)$ is a generalized Meir-Keeler-type $\alpha-\psi$-contraction and the following conditions hold.

(i) If any nondecreasing sequence $\left\{x_{n}\right\}$ in $X$ converges to $v$, then we assume that

$$
x_{n} \sqsubseteq \nu \quad \forall n \in \mathbb{N} .
$$

(ii) There exists $x_{0} \in X$ with $g x_{0} \sqsubseteq f x_{0}$ and $\alpha\left(f x_{0}, f x_{0}\right) \geq$ 1. 
(iii) If $\alpha\left(f x_{n}, f x_{n}\right) \geq 1$ for all $n \in \mathbb{N}$, then $\lim _{n \rightarrow \infty} \alpha\left(f x_{n}, f x_{n}\right) \geq 1$.

Then $f$ and $g$ have a coincidence point in $X$.

Proof. Since $f X \subset g X$ and by (ii), there exists $x_{0} \in X$ with $g x_{0} \sqsubseteq f x_{0}$ and $\alpha\left(f x_{0}, f x_{0}\right) \geq 1$, we can choose $x_{1} \in X$ such that $g x_{1}=f x_{0}$. Since $f$ is $g$-nondecreasing, we have $f x_{0} \sqsubseteq f x_{1}$. In this process, we construct the sequence $\left\{x_{n}\right\}$ recursively as

$$
f x_{n}=g x_{n+1} \quad \forall n \in \mathbb{N} .
$$

Thus, we also conclude that

$$
\begin{aligned}
g x_{0} \sqsubseteq f x_{0} & =g x_{1} \sqsubseteq f x_{1}=g x_{2} \sqsubseteq \cdots \sqsubseteq f x_{n-1} \\
& =g x_{n} \sqsubseteq f x_{n}=g x_{n+1} \sqsubseteq \cdots .
\end{aligned}
$$

If any two consecutive terms in (53) are equal, then the conclusion of the theorem follows. So we may assume that

$$
d\left(f x_{n-1}, f x_{n}\right) \neq 0, \quad \forall n \in \mathbb{N} .
$$

On the other hand, since $f$ is $\alpha$-g-admissible and $\alpha\left(f x_{0}, f x_{0}\right)=\alpha\left(g x_{1}, g x_{1}\right) \geq 1$, we have

$$
\alpha\left(f x_{1}, f x_{1}\right)=\alpha\left(g x_{2}, g x_{2}\right) \geq 1 \text {. }
$$

By continuing this process, we get

$$
\alpha\left(f x_{n}, f x_{n}\right)=\alpha\left(g x_{n+1}, g x_{n+1}\right) \geq 1 \quad \forall n \in \mathbb{N} \cup\{0\} .
$$

By (53), (54), and (56), substituting $x=x_{n}$ and $y=x_{n+1}$ in (50), we have

$$
\begin{aligned}
& d\left(f x_{n}, f x_{n+1}\right) \\
& \leq \alpha\left(f x_{n}, f x_{n}\right) \alpha\left(g x_{n+1}, g x_{n+1}\right) d\left(f x_{n}, f x_{n+1}\right) \\
& <\psi\left(d\left(g x_{n}, g x_{n+1}\right), d\left(g x_{n}, f x_{n}\right), d\left(g x_{n+1}, f x_{n+1}\right),\right. \\
& \left.\quad\left(g x_{n}, f x_{n+1}\right), d\left(g x_{n+1}, f x_{n}\right)\right) \\
& =\psi\left(d\left(f x_{n-1}, f x_{n}\right), d\left(f x_{n-1}, f x_{n}\right), d\left(f x_{n}, f x_{n+1}\right),\right. \\
& \left.d\left(f x_{n-1}, f x_{n+1}\right), d\left(f x_{n}, f x_{n}\right)\right) .
\end{aligned}
$$

If $d\left(f x_{n-1}, f x_{n}\right) \leq d\left(f x_{n}, f x_{n+1}\right)$, then the inequality (57) becomes

$$
\begin{aligned}
& d\left(f x_{n}, f x_{n+1}\right) \\
& <\psi\left(d\left(f x_{n-1}, f x_{n}\right), d\left(f x_{n-1}, f x_{n}\right), d\left(f x_{n}, f x_{n+1}\right)\right. \\
& \left.\quad d\left(f x_{n-1}, f x_{n+1}\right), d\left(f x_{n}, f x_{n}\right)\right) \\
& \leq \psi\left(d\left(f x_{n}, f x_{n+1}\right), d\left(f x_{n}, f x_{n+1}\right), d\left(f x_{n}, f x_{n+1}\right)\right. \\
& \left.\quad 2 d\left(f x_{n}, f x_{n+1}\right), 0\right) \\
& \leq d\left(f x_{n}, f x_{n+1}\right)
\end{aligned}
$$

which implies a contradiction, and we get that $d\left(f x_{n}\right.$, $\left.f x_{n+1}\right)<d\left(f x_{n-1}, f x_{n}\right)$.

From the argument above, we have that the sequence $\left\{d\left(f x_{n}, f x_{n+1}\right)\right\}$ is decreasing, and it must converge to some $\eta \geq 0$, that is,

$$
\lim _{n \rightarrow \infty} d\left(f x_{n}, f x_{n+1}\right)=\eta .
$$

It follow from that (57) and (59), we have

$$
\begin{gathered}
\lim _{n \rightarrow \infty} \psi\left(d\left(f x_{n-1}, f x_{n}\right), d\left(f x_{n-1}, f x_{n}\right), d\left(f x_{n}, f x_{n+1}\right),\right. \\
\left.d\left(f x_{n-1}, f x_{n+1}\right), d\left(f x_{n}, f x_{n}\right)\right)=\eta .
\end{gathered}
$$

Notice that $\eta=\inf \left\{d\left(f x_{n}, f x_{n+1}\right): n \in \mathbb{N} \cup\{0\}\right\}$. We claim that $\eta=0$. Suppose, to the contrary, that $\eta>0$. Since $(f, g)$ is a generalized Meir-Keeler-type $\alpha-\psi$-contraction, corresponding to $\eta$ use, and taking into account the above inequality (60), there exist $\delta>0$ and a natural number $k$ such that

$$
\begin{gathered}
\eta \leq \psi\left(d\left(f x_{k-1}, f x_{k}\right), d\left(f x_{k-1}, f x_{k}\right),\right. \\
d\left(f x_{k}, f x_{k+1}\right), d\left(f x_{k-1}, f x_{k+1}\right), \\
\left.d\left(f x_{k}, f x_{k}\right)\right) \\
<\eta+\delta \Longrightarrow \alpha\left(f x_{k}, f x_{k}\right) \alpha\left(g x_{k+1}, g x_{k+1}\right) \\
\times d\left(f x_{k}, f x_{k+1}\right)<\eta,
\end{gathered}
$$

which implies

$$
\begin{aligned}
d\left(f x_{k}, f x_{k+1}\right) \leq & \alpha\left(f x_{k}, f x_{k}\right) \\
& \times \alpha\left(g x_{k+1}, g x_{k+1}\right) d\left(f x_{k}, f x_{k+1}\right)<\eta .
\end{aligned}
$$

So we get a contradiction, since $\eta=\inf \left\{d\left(f x_{n}, f x_{n+1}\right): n \in\right.$ $\mathbb{N} \cup\{0\}\}$. Thus we have that

$$
\lim _{n \rightarrow \infty} d\left(f x_{n}, f x_{n+1}\right)=0 .
$$

We next claim that $\left\{f x_{n}\right\}$ is a Cauchy sequence, that is, for every $\varepsilon>0$, there exists $n \in \mathbb{N}$ such that if $p, q \geq n$, then $d\left(f x_{p}, f x_{q}\right)<\varepsilon$.

Suppose the above statement is false. Then there exists $\epsilon>$ 0 such that for any $n \in \mathbb{N}$, there are $p_{n}, q_{n} \in \mathbb{N}$ with $p_{n}>q_{n} \geq$ $n$ satisfying

$$
d\left(f x_{q_{n}}, f x_{p_{n}}\right) \geq \epsilon .
$$

Further, corresponding to $q_{n} \geq n$, we can choose $p_{n}$ in such a way that is it the smallest integer with $p_{n}>q_{n} \geq n$ and $d\left(f x_{q_{n}}, f x_{p_{n}}\right) \geq \epsilon$. Therefore $d\left(f x_{q_{n}}, f x_{p_{n}-1}\right)<\epsilon$. Now we have that for all $n \in \mathbb{N}$

$$
\begin{aligned}
\epsilon \leq & d\left(f x_{p_{n}}, f x_{q_{n}}\right) \leq d\left(f x_{p_{n}}, f x_{p_{n}-1}\right) \\
& +d\left(f x_{p_{n}-1}, f x_{q_{n}}\right) \\
< & d\left(f x_{p_{n}}, f x_{p_{n}-1}\right)+\epsilon .
\end{aligned}
$$


Letting $n \rightarrow \infty$, then we get

$$
\lim _{n \rightarrow \infty} d\left(f x_{p_{n}}, f x_{q_{n}}\right)=\epsilon .
$$

On the other hand, we have

$$
\begin{aligned}
d\left(f x_{p_{n}}, f x_{q_{n}}\right) \leq & d\left(f x_{p_{n}}, f x_{p_{n}-1}\right)+d\left(f x_{p_{n}-1}, f x_{q_{n}-1}\right) \\
& +d\left(f x_{q_{n}-1}, f x_{q_{n}}\right), \\
d\left(f x_{p_{n}-1}, f x_{q_{n}-1}\right) \leq & d\left(f x_{p_{n}-1}, f x_{p_{n}}\right)+d\left(f x_{p_{n}}, f x_{q_{n}}\right) \\
& +d\left(f x_{q_{n}}, f x_{q_{n}-1}\right) .
\end{aligned}
$$

Letting $n \rightarrow \infty$, then we get

$$
\lim _{n \rightarrow \infty} d\left(f x_{p_{n}-1}, f x_{q_{n}-1}\right)=\epsilon .
$$

By (53), we have that the elements $g x_{p_{n}}$ and $g x_{q_{n}}$ are comparable. Substituting $x=x_{p_{n}}$ and $y=x_{q_{n}}$ in (50), we have that for all $n \in \mathbb{N}$,

$$
\begin{aligned}
& d(\left.f x_{p_{n}}, f x_{q_{n}}\right) \\
& \leq \alpha\left(f x_{p_{n}}, f x_{p_{n}}\right) \alpha\left(g x_{q_{n}}, g x_{q_{n}}\right) d\left(f x_{p_{n}}, f x_{q_{n}}\right) \\
&<\psi\left(d\left(g x_{p_{n}}, g x_{q_{n}}\right), d\left(g x_{p_{n}}, f x_{p_{n}}\right), d\left(g x_{q_{n}}, f x_{q_{n}}\right),\right. \\
&\left.d\left(g x_{p_{n}}, f x_{q_{n}}\right), d\left(g x_{q_{n}}, f x_{p_{n}}\right)\right) \\
& \leq \psi\left(d\left(f x_{p_{n}-1}, f x_{q_{n}-1}\right), d\left(f x_{p_{n}-1}, f x_{p_{n}}\right),\right. \\
& d\left(f x_{q_{n}-1}, f x_{q_{n}}\right), d\left(f x_{p_{n}-1}, f x_{q_{n}}\right), \\
&\left.\quad d\left(f x_{q_{n}-1}, f x_{p_{n}}\right)\right) \\
& \leq \psi\left(d\left(f x_{p_{n}-1}, f x_{q_{n}-1}\right), d\left(f x_{p_{n}-1}, f x_{p_{n}}\right),\right. \\
& d\left(f x_{q_{n}-1}, f x_{q_{n}}\right), d\left(f x_{p_{n}-1}, f x_{p_{n}}\right)+d\left(f x_{p_{n}}, f x_{q_{n}}\right), \\
&\left.d\left(f x_{q_{n}-1}, f x_{q_{n}}\right)+d\left(f x_{q_{n}}, f x_{p_{n}}\right)\right) .
\end{aligned}
$$

Letting $n \rightarrow \infty$ in (69), then we get

$$
\epsilon<\psi(\epsilon, 0,0, \epsilon, \epsilon) \leq \epsilon,
$$

which implies a contradiction. Thus, $\left\{f x_{n}\right\}$ is a Cauchy sequence.

Since $X$ is complete and $g X$ is closed, there exists $\nu \in X$ such that

$$
\lim _{n \rightarrow \infty} g x_{n}=\lim _{n \rightarrow \infty} f x_{n}=g v .
$$

Since $\alpha$ is continuous in each coordinate and by the condition (iii), we have

$$
\alpha(g \nu, g \nu)=\lim _{n \rightarrow \infty} \alpha\left(f x_{n}, f x_{n}\right) \geq 1 .
$$

Later, we prove that $v$ is a coincidence point of $f$ and $g$. From (53) and (71), we deduce that

$$
g x_{n} \sqsubseteq g v, \quad \forall n \in \mathbb{N} .
$$

By (72) and substituting $x=x_{n}$ and $y=v$ in (50), we have that

$$
\begin{aligned}
& d\left(f x_{n}, f v\right) \leq \alpha\left(f x_{n}, f x_{n}\right) \alpha(g v, g v) d\left(f x_{n}, f v\right) \\
&<\psi\left(d\left(g x_{n}, g \nu\right), d\left(g x_{n}, f x_{n}\right),\right. \\
&\left.\quad d(g v, f v), d\left(g x_{n}, f v\right), d\left(g v, f x_{n}\right)\right) .
\end{aligned}
$$

Taking $n \rightarrow \infty$ in the above inequality, we have

$$
\begin{gathered}
d(g v, f v)<\psi(d(g v, g v), d(g v, g v), \\
\quad d(g v, f v), d(g v, f v), d(g v, g v)) \\
\leq d(g v, f v) .
\end{gathered}
$$

This implies that $g v=f v$. So we complete the proof.

Apply Theorem 15, we are easy to get the following theorem.

Theorem 16. Let $(X, \sqsubseteq, d)$ be a partially ordered complete metric space, and let $f, g: X \rightarrow X$ be such that $f X \subset g X, f$ is $g$-nondecreasing, and $g X$ is closed. Suppose the pair $(f, g)$ is a generalized Meir-Keeler-type $\psi$-contraction and the following conditions hold.

(i) If any nondecreasing sequence $\left\{x_{n}\right\}$ in $X$ converges to $v$, then we assume that

$$
x_{n} \sqsubseteq \nu \quad \forall n \in \mathbb{N} .
$$

(ii) There exists $x_{0} \in X$ with $g x_{0} \sqsubseteq f x_{0}$.

Then $f$ and $g$ have a coincidence point in $X$.

\section{References}

[1] S. Banach, "Sur les operations dans les ensembles abstraits et leur application aux equations integerales," Fundamenta Mathematicae, vol. 3, pp. 133-181, 1922.

[2] L. B. Ćirić, "A generalization of Banach's contraction principle," Proceedings of the American Mathematical Society, vol. 45, pp. 267-273, 1974.

[3] M. Eslamian and A. Abkar, "A fixedpoint theorem for generalized weakly contractive mappings in complete metric space," Italian Journal of Pure and Applied Mathematics. In press.

[4] L. Ćirić, N. Cakić, M. Rajović, and J. S. Ume, "Monotone generalized nonlinear contractions in partially ordered metric spaces," Fixed Point Theory and Applications, vol. 2008, Article ID 131294, 11 pages, 2008.

[5] J. Harjani, B. López, and K. Sadarangani, "Fixed point theorems for weakly $\mathscr{C}$-contractive mappings in ordered metric spaces," Computers \& Mathematics with Applications, vol. 61, no. 4, pp. 790-796, 2011.

[6] J. Harjani, B. López, and K. Sadarangani, "A fixed point theorem for mappings satisfying a contractive condition of rational type on a partially ordered metric space," Abstract and Applied Analysis, vol. 2010, Article ID 190701, 8 pages, 2010. 
[7] J. J. Nieto and R. Rodríguez-López, "Contractive mapping theorems in partially ordered sets and applications to ordinary differential equations," Order, vol. 22, no. 3, pp. 223-239, 2005.

[8] A. C. M. Ran and M. C. B. Reurings, "A fixed point theorem in partially ordered sets and some applications to matrix equations," Proceedings of the American Mathematical Society, vol. 132, no. 5, pp. 1435-1443, 2004.

[9] B. S. Choudhury and A. Kundu, " $(\psi, \alpha, \beta)$-weak contractions in partially ordered metric spaces," Applied Mathematics Letters, vol. 25, no. 1, pp. 6-10, 2012.

[10] A. Meir and E. Keeler, "A theorem on contraction mappings," Journal of Mathematical Analysis and Applications, vol. 28, pp. 326-329, 1969.

[11] B. Samet, C. Vetro, and P. Vetro, "Fixed point theorems for $\alpha$ - $\psi$-contractive type mappings," Nonlinear Analysis. Theory, Methods \& Applications, vol. 75, no. 4, pp. 2154-2165, 2012. 


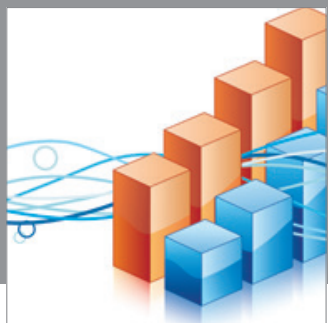

Advances in

Operations Research

mansans

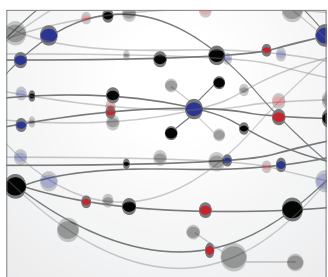

The Scientific World Journal
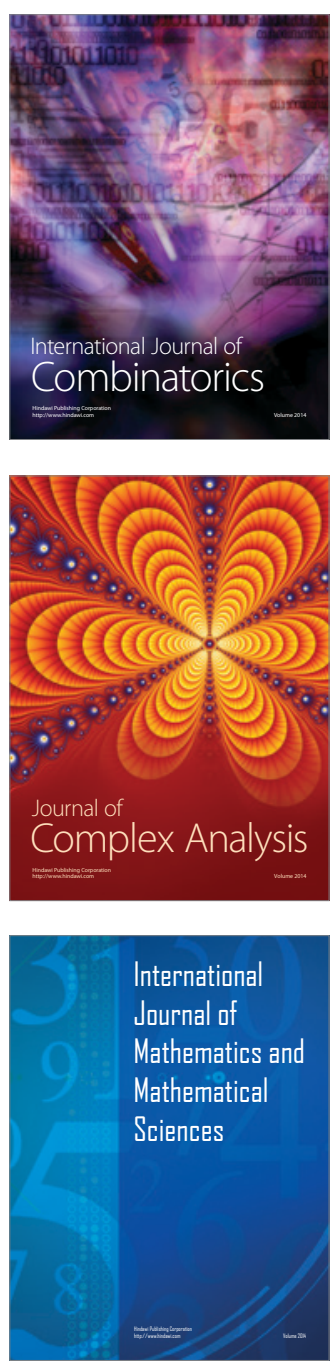
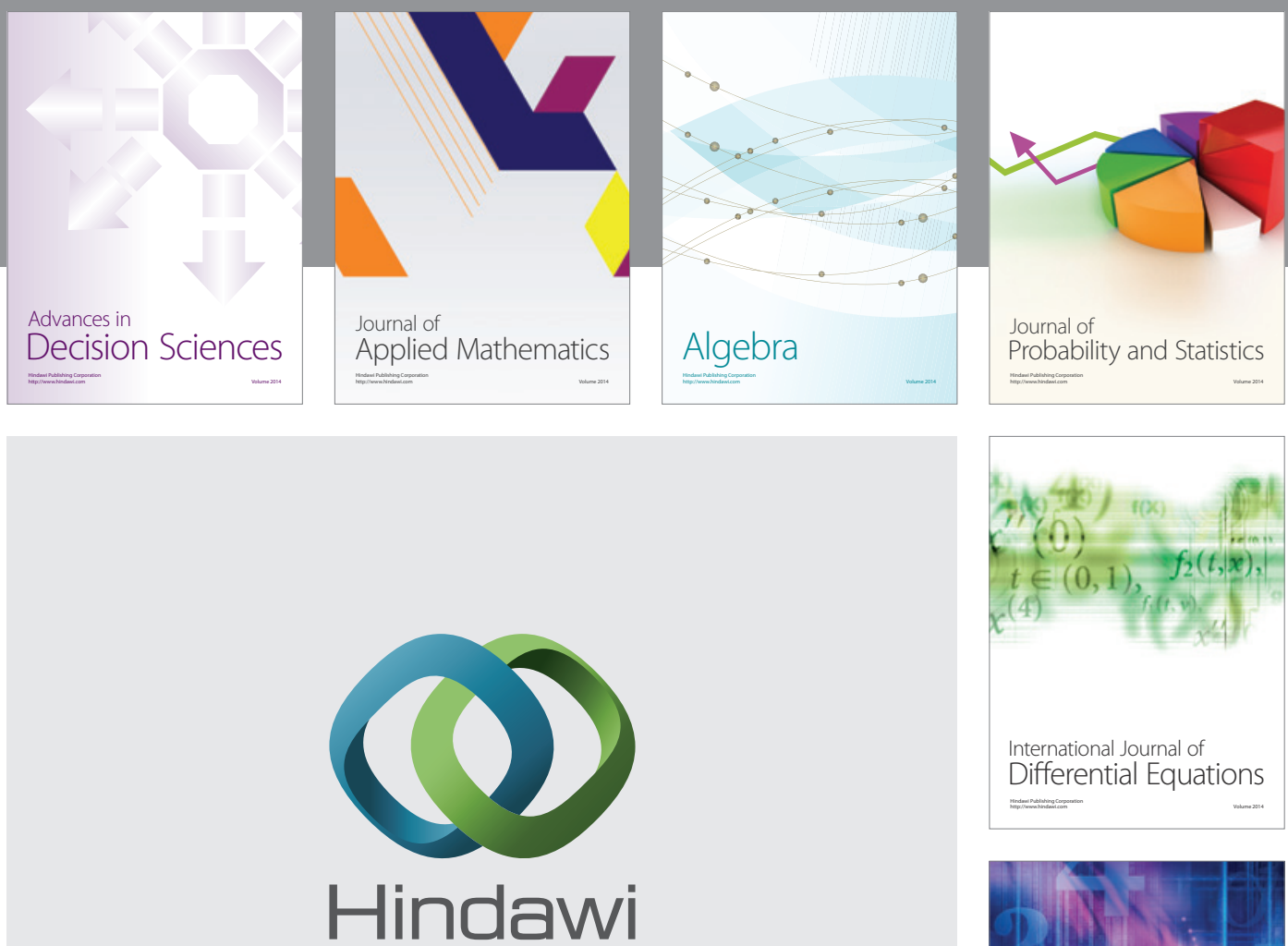

Submit your manuscripts at http://www.hindawi.com
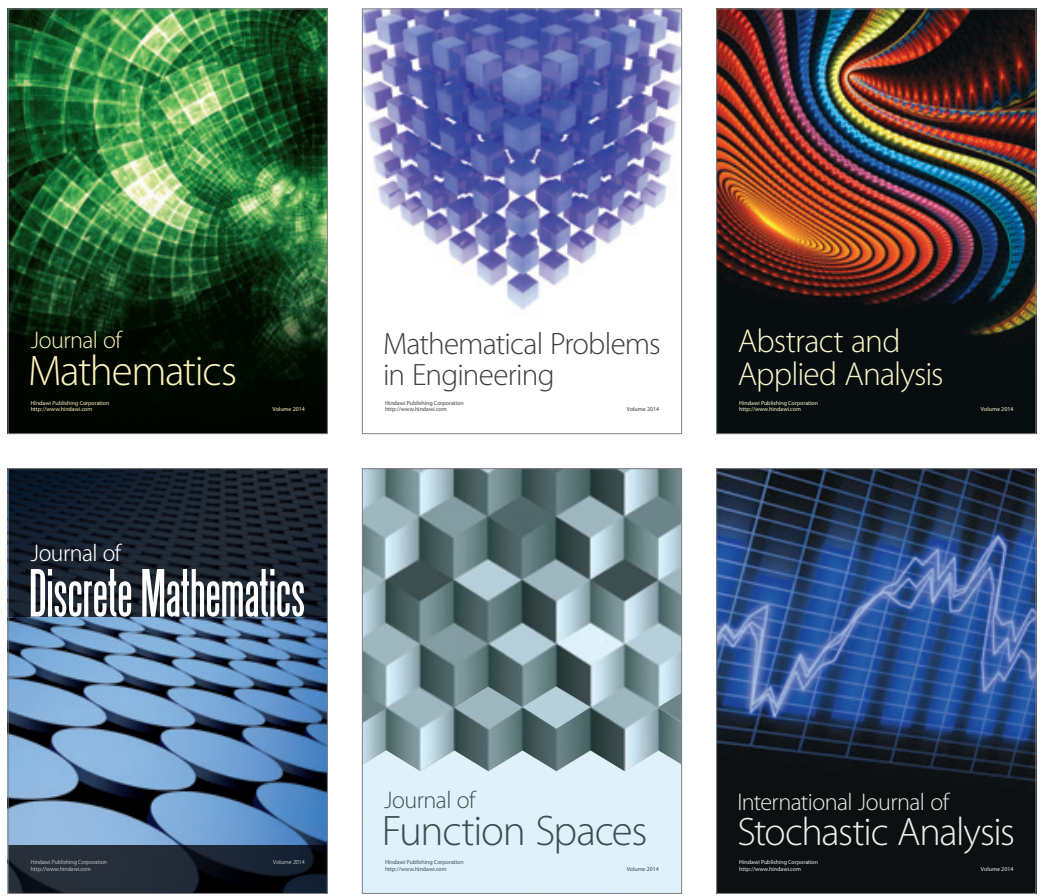

Journal of

Function Spaces

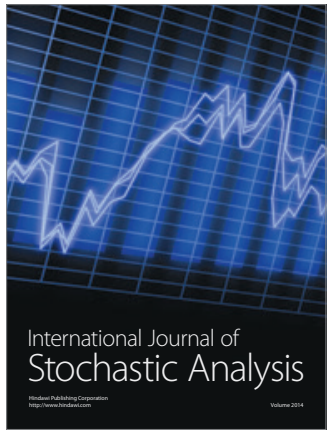

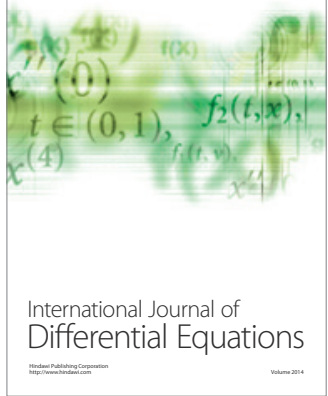
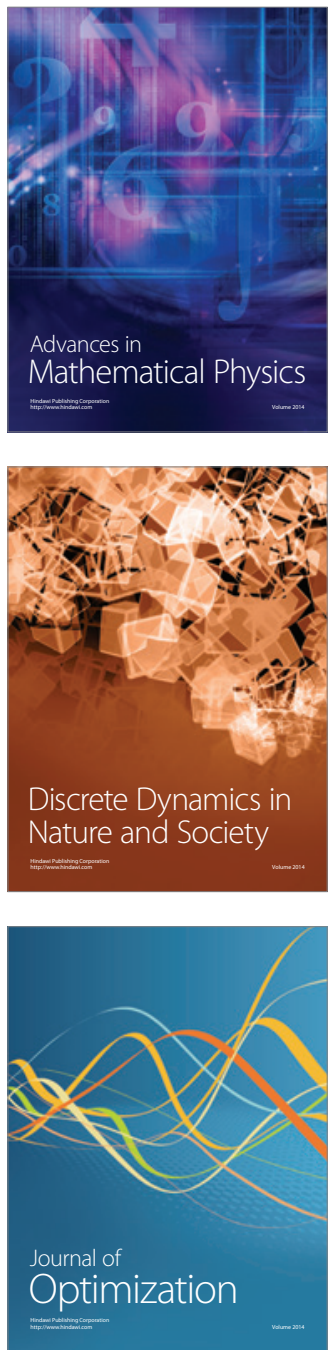